\title{
The association between dairy product consumption and cognitive function in the National Health and Nutrition Examination Survey
}

\author{
Keigan M. Park ${ }^{1 *}$ and Victor L. Fulgoni III $^{2}$ \\ ${ }^{1}$ Dairy Research Institute, 10255 W Higgins Road, Suite 900, Rosemont, IL, 60622, USA \\ ${ }^{2}$ Nutrition Impact, LLC, 9725 D Drive North, Battle Creek, MI 49014, USA \\ (Submitted 20 January 2012 - Final revision received 10 May 2012 - Accepted 30 May 2012 - First published online 24 July 2012)
}

\section{Abstract}

The present cross-sectional study sought to determine the potential relationships between the intake of dairy foods (total dairy products, milk and cheese) and cognitive function through information garnered in the National Health and Nutrition Examination Surveys (1988-94 and 1999-2002). Cognitive measures of vasomotor speed, coding speed and immediate memory recall were assessed from a simple reaction time task (SRTT), symbol-digit substitution test (SDST) and serial digit learning task, respectively, in adults 20-59 years of age. A summation of the percentile rank scores on each of the three tests provided a measure of overall cognitive function. In adults 60 years of age and above, a story recall test and a digit-symbol substitution test (DSST) were utilised to determine cognitive function in an elderly population. The results indicated that cognitive scores for the SRTT were not different between consumers and non-consumers of dairy foods. However, there were associations observed between 20- and 59-year-old consumers of total dairy foods and a higher SDST percentile score $(53 \cdot 2$ (SE 1.3) to 49.4 (SE 2.0)) and a calculated global cognitive percentile score $(53 \cdot 3$ (SE 1.1) to 50.2 (sE 1.4)) compared with nonconsumers. A similar significant association was observed with cheese consumers. In adults over 60 years of age, an association between total dairy product consumption and higher DSST percentile scores ( 51.5 (SE 1.9) to 46.2 (sE 3.0)) was also observed. These findings highlight the need for additional research on how dairy products may affect cognition and by what mechanisms, through its nutrients or other components.

Key words: Dairy foods: Cheese: Cognition: National Health and Nutrition Examination Surveys

There are many environmental factors that may affect the progression of cognitive decline, including inflammatory stress, ecological toxins and a nutritionally poor diet ${ }^{(1)}$. Dietary-mediated effects on neurological function and cognition early in life might lead to the development of frank dementia later in life ${ }^{(2)}$. So far, many hypotheses exist in which the diet may affect neurological functioning; however, there is a current lack of clinical evidence to confirm them. One possible scenario is that alterations in blood flow to the central nervous system (CNS) may result in reduced delivery of vital nutrients or decrease the removal of toxic side products of metabolism $^{(3)}$. In fact, the American Heart Association has indicated the need for additional work on how traditional cardiovascular risk factors, such as hypertension and hypercholesterolaemia, may have an impact on the vascular system and lead to cognitive impairment since these risk factors have been linked to cognitive diseases such as Alzheimer's through epidemiological findings ${ }^{(4)}$. It is likely that the enhanced intake of industrially produced trans-fatty acids, given that consumption has been linked to increases in serum cholesterol, may combine with genetics to negatively affect the CNS. In contrast, the consumption of healthy fats may help to provide the necessary substrates for optimal neuronal health by limiting cholesterol deposition and may work to limit inflammation, both possible causes of cognitive decline $^{(5)}$. Some epidemiological work has already been undertaken with regard to this question, with some results indicating a higher risk of dementia with high-fat diets that include more saturated fat and cholesterol and lower intakes of polyunsaturated fats ${ }^{(6)}$. Still, this evidence is far from conclusive as other studies have shown no effect of dietary fat intakes in the same study cohort ${ }^{(7)}$.

In contrast to the deficits hypothesised by the intake of certain nutrients, it is also likely that optimal neuronal function requires the availability of numerous amino acids, minerals and vitamins ${ }^{(1)}$. For example, an antioxidant-rich diet including higher intakes of vitamins $\mathrm{C}$ and $\mathrm{E}$ has been associated with a lower risk for Alzheimer's disease development ${ }^{(8)}$.

Abbreviations: CNS, central nervous system; DSST, digit-symbol substitution test; NHANES, National Health and Nutrition Examination Survey; SDLT, serial digit learning task; SDST, symbol-digit substitution test.

*Corresponding author: Dr K. M. Park, fax +1 847803 2077, email keigan.park@rosedmi.com 
In addition, the intakes of folate and vitamin $\mathrm{D}$ have also been associated with a reduced risk for Alzheimer's disease and cognitive decline ${ }^{(9-11)}$. Furthermore, some research has been accomplished on the ability of certain dietary patterns to affect cognitive function. In a study by Barberger-Gateau et $a l .^{(12)}$, a diet rich in fruit, vegetables and fish was associated with a decreased risk of dementia and Alzheimer's disease, while others have shown that a Mediterranean diet may reduce the risk for mild cognitive impairment and the development of Alzheimer's disease ${ }^{(13,14)}$. However, these studies fall short of linking specific foods to cognitive function and, in fact, very few studies have examined the ability of individual foods to affect cognitive functioning.

Our current focus has been on examining the ability of dairy foods to affect cognitive health. Possible mechanisms by which dairy products may affect cognitive function were outlined in a recent review by Camfield et al. ${ }^{(15)}$. The authors discussed the potential importance of dairy foods in cognitive health through their hypothesised ability to decrease the risk of the metabolic syndrome, a collection of risk factors that signify an increased risk of CVD and diabetes, and their possible role in stabilising glucose levels. Furthermore, the presence of bioactive peptides, vitamin $\mathrm{B}_{12}$, $\mathrm{Ca}$ and end products of fermentation in dairy foods may play a role in their ability to affect cognitive health. However, even with these hypotheses, there is actually very little evidence to suggest an association between dairy product consumption and cognitive health. A recent review outlined the limited findings of several published studies examining the ability of dairy food intake to affect cognitive function ${ }^{(16)}$. The review concluded that a lower consumption of dairy products was generally found to increase the risk for vascular dementia ${ }^{(16)}$. This was more pronounced for low-fat dairy products, as some evidence indicated that the consumption of full-fat dairy products may be linked with cognitive deficits in the elderly. However, the data were specific to several individual population groups and therefore not very informative with regard to the population as a whole. In addition, since early defects can lead to long-term shortfalls in cognition, we felt it was important to study not only the effects of dietary factors on cognition in the elderly but also in a younger population. We hypothesised that the relationship with dairy products would be conserved for both milk and cheese, as both dairy products contribute similar nutrients to the diet. In order to confirm or refute our hypothesis, we sought a nationally representative database that afforded a large enough dataset to examine possible associations in adults and in the elderly with different facets of cognitive health. In the present study, the National Health and Nutrition Examination Survey (NHANES) was utilised to crosssectionally assess the potential relationship between dairy product consumption and cognitive measures.

\section{Experimental methods}

The present study utilised data from the NHANES database, compiled by the Centers for Disease Control and Prevention. Ethical approval of the interviews and physical examinations was obtained from the NHANES Institutional Review Board and documented consent was obtained from each participant. The 1988-94 NHANES dataset included three cognitive tests for individuals 20-59 years of age as a single, computerised test battery called the Neuropsychological Evaluation System ${ }^{(17,18)}$. The battery included the simple reaction time task, which measures visuomotor speed, by assessing an individual's response time to a random presentation of specific visual symbols over a number of trials. It also included the symbol-digit substitution test (SDST), which measures information-processing speed, concentration and motor control by having the subject memorise nine symbols matched to integers 1 to 9. The symbols are then shown in a random order and the subject is responsible for providing the corresponding digit as quickly as possible from memory. The last test in this battery was the serial digit learning task (SDLT), which measures learning and recall by having the subject memorise a predefined sequence of numbers, and subsequently testing their ability to repeat the sequence consecutively with as few errors as possible. To maintain consistency, scores were converted to a percentile score for each subject, with a higher percentile score representing a better score on the cognitive assessments. By combining the results from each of the three primary tests included in NHANES III, a measure of global cognitive function was obtained. This was accomplished, as previously demonstrated by Zhang et al. ${ }^{(19)}$, by summing the percentile rank scores achieved on each of the three tests. In this same NHANES survey, a story recall was utilised as the only test of cognitive endpoints (attention and delayed verbal memory) in adults 60 years of age and older $(60+)$. In this test, subjects are assessed on their ability to remember details from a story presented to them by the interviewer. Again, scores were converted to a percentile, with a higher percentile indicating a better cognitive score. In order to provide additional data for the $60+$ age group, we also utilised a later dataset (NHANES 1999-2002) which contained the digit-symbol substitution test (DSST) of response speed, sustained attention, visual spatial skills, and associative learning and memory in adults over 60 years. The DSST requires that the subject correctly codes a series of symbols in $2 \mathrm{~min}$. This exercise is thought to be a sensitive measure of dementia, and has been administered in the National Institute on Aging's Health ABC study ${ }^{(20-22)}$.

An in-person $24 \mathrm{~h}$ dietary recall was obtained from participants in the NHANES 1988-94 and 1999-2002 datasets ${ }^{(23,24)}$. Dairy consumption from all sources (including both low fat and full fat) was defined as (1) total dairy products (including milk, cheese and yogurt), (2) dairy servings from milk and (3) dairy servings from cheese (yogurt intake was too low to be analysed separately). Dairy product consumers were defined as those consuming at least one serving of dairy products (one cup equivalent) per recall, whereas non-consumers were defined as indicating no servings of dairy products consumed. Analyses were conducted for two separate age groups in NHANES 1988-94, namely adults $20-59$ years $(n 4355)$ and $60+(n$ 4282), while only adults $60+(n$ 2189) were analysed in NHANES 1999-2002. All analyses were adjusted for major covariates that have been previously shown to affect public health. These included sex, ethnicity, age, smoking 
status, energy intake $(\mathrm{kJ}), \mathrm{BMI}\left(\mathrm{kg} / \mathrm{m}^{2}\right)$, poverty income ratio, intakes of saturated fat $(\mathrm{g})$, monounsaturated fat $(\mathrm{g})$, polyunsaturated fat $(\mathrm{g})$, cholesterol $(\mathrm{mg})$, carbohydrate $(\mathrm{g})$ and protein $(\mathrm{g})$. Unreliable records (e.g. energy intake that was unreasonable), pregnant or lactating females, any person not taking one of the cognitive tests and any person with missing values for any of the covariates were excluded from the analysis. Additionally, all analyses were conducted with appropriate sample weighting for analysing the NHANES data.

ANOVA, adjusting for the complex sample design of the NHANES, were used to determine whether there was an association between dairy product intake variables and cognitive parameters. ANOVA was used to ascertain whether cognitive measures were different among consumers and nonconsumers of dairy product intake variables. Additionally, subjects were separated into quintiles of dairy product intake variables (by age group) and ANOVA was used to assess a $P$-for-trend of the relationship of dairy product intake with cognitive measures. The first quintile included non-consumers and consumers were split into four additional quartiles of dairy product consumption based on cup equivalents of dairy foods consumed. Cut-offs for total dairy product consumption in 20-59-year-olds in NHANES 1988-94 were 0, $0.1-0.62,0.63-1.32,1.33-2.38$ and $>2.38$ cup equivalents, comparable data for milk were $0,0 \cdot 1-0.23,0.24-0.76$, $0.77-1.7$ and $>1.7$ cup equivalents and for cheese were 0 , $0 \cdot 1-0.37,0.38-0.74,0.75-1.34$ and $>1.34$ cup equivalents. Cut-offs for total dairy product consumption in $60+$ in NHANES 1988-94 were $0,0 \cdot 1-0.50,0.51-1 \cdot 09,1 \cdot 10-2 \cdot 00$ and $>2.01$ cup equivalents, for milk were $0,0 \cdot 1-0 \cdot 39$, $0.40-0.93,0.94-1.71$ and $>1.72$ cup equivalents and for cheese were $0,0 \cdot 1-0 \cdot 21,0 \cdot 22-0.42,0.43-0.81$ and $>0.82$ cup equivalents. Cut-offs for total dairy product consumption in $60+$ in NHANES $1999-2002$ were $0,0 \cdot 1-0 \cdot 52,0 \cdot 53-1 \cdot 13$, $1.14-1.99$ and $>2.00$ cup equivalents, for milk were 0 , $0 \cdot 1-0.31,0.32-0.82,0.83-1.50$ and $>1.51$ cup equivalents and for cheese were $0,0 \cdot 1-0 \cdot 18,0 \cdot 19-0 \cdot 47,0 \cdot 48-0.87$ and $>0 \cdot 88$ cup equivalents.

\section{Results}

Percentile scores for the simple reaction time task, SDLT and SDST in adults 20-59 years of age were not different for consumers and non-consumers of total dairy products (Table 1). In contrast, in the $60+$ age group, consumers of total dairy products were associated with higher percentile scores on the short-term memory measure (story recall) when compared with non-consumers (49.6 (SE 0.7) $v$. 43.7 (SE 1.3) for a combined score of two stories, $P<0 \cdot 0001)$. Comparing consumers and non-consumers, milk consumption was not related to any cognitive measures in either age group (Table 2 ). In the $60+$ age group, consumers of cheese (Table 3 ) had higher story recall percentile scores when compared with non-consumers (51.9 (SE 0.9) $v .47 \cdot 3$ (SE 0.7 ) for a combined score of two stories, $P<0.0001$ ). Additionally, there was some support that cheese consumers had higher DSST percentile scores when examining individuals $60+$ in the NHANES 1999-2002 dataset (52.0 (SE 0.8) v. 48.4 (SE 1.2), $P=0.0221)$.

When looking at quintiles of consumption (non-consumers and quartiles of dairy product consumption) in NHANES 1988-94 (ages 20-59 years), there was a $P$-for-trend for SDST percentiles as total dairy product intake increased (49.4 (SE 2.0) to 53.2 (sE 1.3) in non-consumers and the highest quartile of dairy product intake, respectively, $P<0.002$; Table 4). This led to a significant association of total dairy product intake with the calculated global score for cognitive performance in the 20-59-year-olds (50.2 (SE 1.4) to 53.3 (SE 1.1) in non-consumers and the highest quartile of dairy product intake, respectively, $P<0 \cdot 0207)$. Additionally, there was a significant $P$-for-trend with DSST percentile scores in individuals $60+$ in NHANES 1999-02 (46.2 (se 3.0) to $51 \cdot 5$ (SE 1.9) in nonconsumers and the highest quartile of dairy intake, respectively, $P<0.009$ ). In agreement with the previous analysis, milk consumption was not related to any cognitive measure when looking at quintile analyses for either age group (Table 5). In contrast, cheese consumption was related to SDST percentiles in the 20-59-year-olds (49.0 (SE 0.9) to $55 \cdot 2$ (SE 1.2) in non-consumers and the highest quintiles of

Table 1. Impact of total dairy product servings (some $v$. none) on cognitive measures in the National Health and Nutrition Examination Survey (NHANES) 1988-94 (NHIII) and NHANES 1999-2002 (NH 99-02)* (Least square mean (LSM) values with their standard errors; number of participants)

\begin{tabular}{|c|c|c|c|c|c|c|c|c|c|}
\hline \multirow[b]{2}{*}{ Dataset } & \multirow[b]{2}{*}{ Age (years) } & \multirow[b]{2}{*}{ Test } & \multicolumn{3}{|c|}{ Non-consumers } & \multicolumn{3}{|c|}{ Consumers } & \multirow[b]{2}{*}{$P$} \\
\hline & & & $n$ & LSM & SE & $n$ & LSM & SE & \\
\hline NHIII & $20-59$ & SDLT & 526 & 49.51 & 2.05 & 3829 & $50 \cdot 42$ & 0.69 & 0.6498 \\
\hline NHIII & $20-59$ & SDST & 526 & $49 \cdot 36$ & $2 \cdot 00$ & 3829 & $50 \cdot 56$ & 0.68 & 0.5164 \\
\hline NHIII & $20-59$ & SRTT & 526 & $51 \cdot 80$ & $1 \cdot 87$ & 3829 & $49 \cdot 97$ & 0.86 & 0.3064 \\
\hline NHIII & $20-59$ & 20-59 All & 526 & $50 \cdot 22$ & 1.41 & 3829 & $50 \cdot 32$ & 0.56 & 0.9474 \\
\hline NHIII & $60+$ & SR-1 & 478 & 44.50 & 1.41 & 3804 & $49 \cdot 87$ & 0.75 & 0.0003 \\
\hline NHIII & $60+$ & SR-2 & 478 & $42 \cdot 80$ & 1.42 & 3804 & $49 \cdot 41$ & 0.70 & 0.0000 \\
\hline NHIII & $60+$ & SR-All & 478 & 43.65 & $1 \cdot 33$ & 3804 & $49 \cdot 64$ & 0.71 & 0.0000 \\
\hline NH 99-02 & $60+$ & DSST & 208 & $46 \cdot 16$ & 2.95 & 1981 & $50 \cdot 63$ & 0.65 & 0.1149 \\
\hline
\end{tabular}

SDLT, serial digit learning task; SDST, symbol-digit substitution test; SRTT, simple reaction time task; SR-1, story recall one; SR-2, story recall two; DSST, digit-symbol substitution test.

* Data were adjusted for sex, ethnicity, age, smoking status, energy intake $(\mathrm{kJ}), \mathrm{BMI}\left(\mathrm{kg} / \mathrm{m}^{2}\right)$, poverty:income ratio, intakes of saturated fat $(\mathrm{g})$, monounsaturated fat $(\mathrm{g})$, polyunsaturated fat $(\mathrm{g})$, cholesterol $(\mathrm{mg})$, carbohydrate $(\mathrm{g})$ and protein $(\mathrm{g})$. 
Table 2. Impact of milk servings (some $v$. none) on cognitive measures in the National Health and Nutrition Examination Survey (NHANES) 1988-94 (NHIII) and NHANES 1999-2002 (NH 99-02)*

(Least square mean (LSM) values with their standard errors; number of participants)

\begin{tabular}{|c|c|c|c|c|c|c|c|c|c|}
\hline \multirow[b]{2}{*}{ Dataset } & \multirow[b]{2}{*}{ Age (years) } & \multirow[b]{2}{*}{ Test } & \multicolumn{3}{|c|}{ Non-consumers } & \multicolumn{3}{|c|}{ Consumers } & \multirow[b]{2}{*}{$P$} \\
\hline & & & $n$ & LSM & $\mathrm{SE}$ & $n$ & LSM & SE & \\
\hline $\mathrm{NHIII}$ & $20-59$ & SDLT & 1072 & $50 \cdot 28$ & 1.43 & 3283 & $50 \cdot 36$ & 0.70 & 0.9524 \\
\hline NHIII & $20-59$ & SDST & 1072 & $50 \cdot 74$ & 1.46 & 3283 & 50.39 & 0.67 & 0.7996 \\
\hline NHIII & $20-59$ & SRTT & 1072 & $51 \cdot 35$ & 1.56 & 3283 & 49.80 & 0.97 & 0.3811 \\
\hline NHIII & $20-59$ & 20-59 All & 1072 & $50 \cdot 79$ & $1 \cdot 11$ & 3283 & $50 \cdot 19$ & 0.59 & 0.6114 \\
\hline NHIII & $60+$ & SR-1 & 674 & 47.67 & 1.48 & 3608 & 49.67 & 0.76 & 0.1813 \\
\hline NHIII & $60+$ & SR-2 & 674 & $47 \cdot 23$ & 1.55 & 3608 & 49.08 & 0.69 & 0.2407 \\
\hline NHIII & $60+$ & SR-All & 674 & 47.45 & 1.44 & 3608 & 49.37 & 0.70 & 0.1841 \\
\hline NH 99-02 & $60+$ & DSST & 386 & $47 \cdot 87$ & $1 \cdot 81$ & 1803 & $50 \cdot 74$ & 0.75 & 0.1402 \\
\hline
\end{tabular}

SDLT, serial digit learning task; SDST, symbol-digit substitution test; SRTT, simple reaction time task; SR-1, story recall one; SR-2, story recall two; DSST, digit-symbol substitution test.

* Data were adjusted for sex, ethnicity, age, smoking status, energy intake $(\mathrm{kJ}), \mathrm{BMI}\left(\mathrm{kg} / \mathrm{m}^{2}\right)$, poverty:income ratio, intakes of saturated fat $(\mathrm{g})$, monounsaturated fat $(\mathrm{g})$, polyunsaturated fat $(\mathrm{g})$, cholesterol $(\mathrm{mg})$, carbohydrate $(\mathrm{g})$ and protein $(\mathrm{g})$.

dairy product intake, respectively, $P<0.001$; Table 6) and short-term memory in the $60+$ age group via the average of story recalls $(47 \cdot 3$ (SE 0.7$)$ to $50 \cdot 5$ (SE 1.8 ) in non-consumers and the highest quartile of dairy product intake, respectively, $P<0 \cdot 0003)$. With an additional modest effect on the SDLT, cheese consumption was also related to a global score for cognitive performance in the 20-59-year-olds (49.9) (SE 0.7 ) to 52.9 (SE 0.9) in non-consumers and the highest quartile of dairy product intake, respectively, $P<0 \cdot 006$ ).

\section{Discussion}

The results of the present cross-sectional analysis indicated that there are some associations between dairy product consumption and cognitive function that deserve further research. In particular, total dairy product consumption in consumers $v$. non-consumers was associated with a higher story recall percentile scores in adults $60+$, which was also significant for cheese intake but not for milk consumption. In addition, when looking across dairy product consumption quintiles in 20-59-year-olds, there was an association with the SDST for both total dairy product and cheese consumption, which drove an association for the calculated global cognitive percentile score. However, again, there was no association with milk consumption. To the extent possible, the data were adjusted for numerous covariates that may have had an effect on cognitive performance (see the Methods section). These adjustments, plus the size of the NHANES dataset and its applicability to the national population, provide some confidence that the associations presented may be the real effects of dairy product intake. Nonetheless, NHANES data are cross-sectional, resulting from observational information and, as such, cause and effect cannot be concluded. In addition, some dietary factors which may have a significant impact on cognition, such as total fruit and vegetable intake, $n-3$ fatty acids, and alcohol consumption were not specifically addressed in the present analysis. Further research with a database containing more cognitive assessments and a greater dietary assessment accuracy is required before the association can be completely confirmed and clinical research is needed before causality can be assigned.

Similar trends were observed with the SDST and DSST, which are similar but not identical tests of coding speed, in both the 20-59 and $60+$ age groups, indicating that more research on the ability of dairy food to affect this facet of cognitive function is needed. However, the absence of the other

Table 3. Impact of cheese servings (some $v$. none) on cognitive measures in the National Health and Nutrition Examination Survey (NHANES) 1988-94 (NHIII) and NHANES 1999-2002 (NH 99-02)*

(Least square mean (LSM) values with their standard errors; number of participants)

\begin{tabular}{|c|c|c|c|c|c|c|c|c|c|}
\hline \multirow[b]{2}{*}{ Dataset } & \multirow[b]{2}{*}{ Age (years) } & \multirow[b]{2}{*}{ Test } & \multicolumn{3}{|c|}{ Non-consumers } & \multicolumn{3}{|c|}{ Consumers } & \multirow[b]{2}{*}{$P$} \\
\hline & & & $n$ & LSM & SE & $n$ & LSM & SE & \\
\hline $\mathrm{NHIII}$ & $20-59$ & SDLT & 1914 & $49 \cdot 69$ & 1.00 & 2441 & $50 \cdot 75$ & 0.82 & 0.3716 \\
\hline NHIII & $20-59$ & SDST & 1914 & 49.01 & 0.94 & 2441 & 51.35 & 0.89 & 0.0538 \\
\hline $\mathrm{NHIII}$ & $20-59$ & SRTT & 1914 & 50.91 & $1 \cdot 15$ & 2441 & 49.63 & 0.92 & 0.2517 \\
\hline $\mathrm{NHIII}$ & $20-59$ & 20-59 All & 1914 & 49.87 & 0.72 & 2441 & 50.57 & 0.68 & 0.4201 \\
\hline $\mathrm{NHIII}$ & $60+$ & SR-1 & 2774 & $47 \cdot 61$ & 0.85 & 1508 & $52 \cdot 10$ & 1.02 & 0.0004 \\
\hline $\mathrm{NHIII}$ & $60+$ & SR-2 & 2774 & 47.01 & 0.70 & 1508 & 51.57 & 0.90 & 0.0000 \\
\hline NHIII & $20-59$ & SR-All & 2774 & $47 \cdot 31$ & 0.74 & 1508 & 51.84 & 0.94 & 0.0000 \\
\hline NH 99-02 & $60+$ & DSST & 1127 & 48.39 & $1 \cdot 22$ & 1062 & 51.98 & 0.80 & 0.0221 \\
\hline
\end{tabular}

SDLT, serial digit learning task; SDST, symbol-digit substitution test; SRTT, simple reaction time task; SR-1, story recall one; SR-2, story recall two; DSST, digit-symbol substitution test.

*Data were adjusted for sex, ethnicity, age, smoking status, energy intake $(\mathrm{kJ}), \mathrm{BMl}\left(\mathrm{kg} / \mathrm{m}^{2}\right)$, poverty:income ratio, intakes of saturated fat (g), monounsaturated fat $(\mathrm{g})$, polyunsaturated fat $(\mathrm{g})$, cholesterol $(\mathrm{mg})$, carbohydrate $(\mathrm{g})$ and protein $(\mathrm{g})$. 
Table 4. Impact of total dairy servings (quartiles of intake) on cognitive measures in the National Health and Nutrition Examination Survey (NHANES) 1988-94 (NHIII) and NHANES 1999-2002 (NH 99-02)*

(Least square mean (LSM) values with their standard errors)

\begin{tabular}{|c|c|c|c|c|c|c|c|c|c|c|c|c|c|}
\hline \multirow[b]{3}{*}{ Dataset } & \multirow[b]{3}{*}{ Age (years) } & \multirow[b]{3}{*}{ Test } & & & \multicolumn{8}{|c|}{ Quartiles of intake } & \multirow{3}{*}{$\begin{array}{c}P \\
\text { Quartile } \\
\text { trend }\end{array}$} \\
\hline & & & \multicolumn{2}{|c|}{ Non-consumers } & \multicolumn{2}{|c|}{ Q1 } & \multicolumn{2}{|c|}{ Q2 } & \multicolumn{2}{|c|}{ Q3 } & \multicolumn{2}{|c|}{ Q4 } & \\
\hline & & & LSM & SE & LSM & SE & LSM & SE & LSM & SE & LSM & SE & \\
\hline $\mathrm{NHIII}$ & $20-59$ & SDLT & 49.51 & 2.05 & $48 \cdot 88$ & $1 \cdot 14$ & $50 \cdot 27$ & 1.29 & 49.58 & $1 \cdot 14$ & $53 \cdot 18$ & 1.32 & 0.1259 \\
\hline NHIII & $20-59$ & SDST & $49 \cdot 36$ & 2.00 & 48.21 & 1.05 & 50.45 & 0.93 & 50.57 & 1.29 & 53.24 & 1.27 & 0.0020 \\
\hline $\mathrm{NHIII}$ & $20-59$ & SRTT & $51 \cdot 80$ & 1.87 & 49.02 & 1.72 & 50.51 & 1.90 & 46.98 & 1.22 & $53 \cdot 56$ & 1.71 & 0.5149 \\
\hline NHIII & $20-59$ & 20-59 All & $50 \cdot 22$ & 1.41 & $48 \cdot 70$ & 0.96 & $50 \cdot 41$ & 0.93 & 49.04 & 0.79 & $53 \cdot 33$ & $1 \cdot 11$ & 0.0207 \\
\hline NHIII & $60+$ & SR-1 & 44.50 & 1.41 & 50.19 & 1.45 & 48.03 & 1.03 & $52 \cdot 42$ & 1.30 & 48.98 & 1.33 & 0.1110 \\
\hline $\mathrm{NHIII}$ & $60+$ & SR-2 & $42 \cdot 80$ & 1.42 & $49 \cdot 76$ & 1.41 & $49 \cdot 24$ & $1 \cdot 27$ & $50 \cdot 64$ & 1.22 & 48.01 & $1 \cdot 26$ & 0.1673 \\
\hline NHIII & $60+$ & SR-All & 43.65 & 1.33 & 49.98 & 1.38 & 48.64 & 1.09 & 51.53 & 1.20 & 48.49 & 1.24 & 0.1181 \\
\hline NH 99-02 & $60+$ & DSST & $46 \cdot 16$ & 2.95 & $48 \cdot 27$ & $1 \cdot 11$ & $50 \cdot 26$ & 1.04 & 52.65 & 0.96 & 51.48 & 1.88 & 0.0090 \\
\hline
\end{tabular}

SDLT, serial digit learning task; SDST, symbol-digit substitution test; SRTT, simple reaction time task; SR-1, story recall one; SR-2, story recall two; DSST, digit-symbol substitution test.

* Data were adjusted for sex, ethnicity, age, smoking status, energy intake (kJ), BMI $\left(\mathrm{kg} / \mathrm{m}^{2}\right)$, poverty:income ratio, intakes of saturated fat (g), monounsaturated fat (g), polyunsaturated fat $(\mathrm{g})$, cholesterol $(\mathrm{mg})$, carbohydrate $(\mathrm{g})$ and protein $(\mathrm{g})$.

cognitive tests (SDLT, story recall) in both age groups makes it difficult to understand whether these associations, or the absence of an association, would hold true throughout the lifespan. It is possible that dairy product consumption may be having acute effects on cognition or dairy product intake may be having long-term effects that are manifested in different cognitive examinations. In addition, due to the low number of non-dairy product consumers, it was not possible to separate the age groups into further population segments. It would be interesting to examine a dataset that allowed for these measures, as the effects of dairy products may be different depending on the consumer's age. Prospective analysis is needed to determine whether long-term exposure to dairy products may decrease the risk for cognitive impairment, and clinical assessments are necessary to determine whether acute exposures to dairy products are enough to elicit shortterm cognitive changes.

It is an interesting finding that cheese consumption may be associated with better cognitive functioning, since previous studies have indicated that the consumption of full-fat dairy products may be associated with negative cognitive outcomes ${ }^{(16)}$. These findings have typically been explained by pointing to the saturated fat content of full-fat dairy products. However, in a study published by Rahman et al. ${ }^{(25)}$, a link between cheese consumption and improved cognition scores was also found. The authors indicated that the nutrients found in dairy products, possibly vitamin A and/or individual dairy fats, may be playing a role in this association. These two divergent findings indicate the need for further research on this topic. These future studies should be designed in such a way that cheese consumption is controlled and the nutrients found in cheese are tracked from both cheese and noncheese sources. This is very important with respect to foods containing saturated fat, since they are composed of many different types of fatty acids, both saturated and unsaturated, which may have significantly diverse health effects ${ }^{(26)}$. In addition, effects of individual nutrients being contributed from cheese such as vitamin A, may not have the same effect in individuals who are already consuming adequate levels of vitamin A from other sources. With careful control,

Table 5. Impact of milk servings (quartiles of intake) on cognitive measures in the National Health and Nutrition Examination Survey (NHANES) 1988-94 (NHIII) and NHANES 1999-2002 (NH 99-02)*

(Least square mean (LSM) values with their standard errors)

\begin{tabular}{|c|c|c|c|c|c|c|c|c|c|c|c|c|c|}
\hline \multirow[b]{3}{*}{ Dataset } & \multirow[b]{3}{*}{ Age (years) } & \multirow[b]{3}{*}{ Test } & & & \multicolumn{8}{|c|}{ Quartiles of intake } & \multirow{3}{*}{$\begin{array}{c}P \\
\text { Quartile } \\
\text { trend }\end{array}$} \\
\hline & & & \multicolumn{2}{|c|}{ Non-consumers } & \multicolumn{2}{|c|}{ Q1 } & \multicolumn{2}{|c|}{ Q2 } & \multicolumn{2}{|c|}{ Q3 } & \multicolumn{2}{|c|}{ Q4 } & \\
\hline & & & LSM & SE & LSM & SE & LSM & SE & LSM & SE & LSM & SE & \\
\hline $\mathrm{NHIII}$ & $20-59$ & SDLT & $50 \cdot 28$ & 1.43 & 47.96 & 1.23 & $51 \cdot 23$ & 1.41 & $50 \cdot 86$ & $1 \cdot 19$ & 51.55 & $1 \cdot 26$ & 0.2477 \\
\hline NHIII & $20-59$ & SDST & $50 \cdot 74$ & 1.46 & $50 \cdot 24$ & $1 \cdot 10$ & $50 \cdot 66$ & $1 \cdot 23$ & 49.93 & 1.27 & $50 \cdot 76$ & 1.38 & 0.9271 \\
\hline NHIII & $20-59$ & SRTT & 51.35 & 1.56 & 48.04 & 1.36 & 50.66 & 1.74 & 49.54 & 1.78 & 51.08 & 1.97 & 0.8511 \\
\hline $\mathrm{NHIII}$ & $20-59$ & 20-59 All & $50 \cdot 79$ & $1 \cdot 11$ & 48.75 & 0.81 & 50.85 & 1.08 & $50 \cdot 11$ & 1.00 & $51 \cdot 13$ & 1.17 & 0.5061 \\
\hline NHIII & $60+$ & SR-1 & $47 \cdot 67$ & 1.48 & $49 \cdot 12$ & 1.31 & $49 \cdot 66$ & 0.96 & $50 \cdot 14$ & $1 \cdot 26$ & 49.81 & 1.25 & 0.1534 \\
\hline NHIII & $60+$ & SR-2 & $47 \cdot 23$ & 1.55 & 48.65 & 1.29 & $49 \cdot 41$ & $1 \cdot 18$ & 49.80 & 1.36 & 48.43 & 1.05 & 0.3456 \\
\hline NHIII & $60+$ & SR-All & 47.45 & 1.44 & 48.88 & 1.23 & 49.54 & 1.02 & 49.97 & 1.26 & $49 \cdot 12$ & $1 \cdot 10$ & 0.2018 \\
\hline NH 99-02 & $60+$ & DSST & $47 \cdot 87$ & 1.81 & 48.98 & 1.34 & 51.22 & 1.08 & 53.33 & $1 \cdot 15$ & 49.50 & 1.83 & 0.1576 \\
\hline
\end{tabular}

SDLT, serial digit learning task; SDST, symbol-digit substitution test; SRTT, simple reaction time task; SR-1, story recall one; SR-2, story recall two; DSST, digit-symbol substitution test.

* Data were adjusted for sex, ethnicity, age, smoking status, energy intake (kJ), BMI ( $\left.\mathrm{kg} / \mathrm{m}^{2}\right)$, poverty:income ratio, intakes of saturated fat (g), monounsaturated fat (g), polyunsaturated fat $(\mathrm{g})$, cholesterol $(\mathrm{mg})$, carbohydrate $(\mathrm{g})$ and protein $(\mathrm{g})$. 
Table 6. Impact of cheese servings (quartiles of intake) on cognitive measures in the National Health and Nutrition Examination Survey (NHANES) 1988-94 (NHIII) and NHANES 1999-2002 (NH 99-02)*

(Least square mean (LSM) values with their standard errors)

\begin{tabular}{|c|c|c|c|c|c|c|c|c|c|c|c|c|c|}
\hline \multirow[b]{3}{*}{ Dataset } & \multirow[b]{3}{*}{ Age (years) } & \multirow[b]{3}{*}{ Test } & & & \multicolumn{8}{|c|}{ Quartiles of intake } & \multirow{3}{*}{$\begin{array}{c}P \\
\text { Quartile } \\
\text { trend }\end{array}$} \\
\hline & & & \multicolumn{2}{|c|}{ Non-consumers } & \multicolumn{2}{|c|}{ Q1 } & \multicolumn{2}{|c|}{ Q2 } & \multicolumn{2}{|c|}{ Q3 } & \multicolumn{2}{|c|}{ Q4 } & \\
\hline & & & LSM & SE & LSM & SE & LSM & SE & LSM & SE & LSM & SE & \\
\hline $\mathrm{NHIII}$ & $20-59$ & SDLT & $49 \cdot 69$ & 1.00 & $48 \cdot 32$ & 1.50 & $50 \cdot 72$ & 1.75 & 51.42 & 1.84 & $52 \cdot 71$ & $1 \cdot 37$ & 0.0391 \\
\hline NHIII & $20-59$ & SDST & 49.01 & 0.94 & $50 \cdot 29$ & 1.65 & $50 \cdot 39$ & 1.38 & $50 \cdot 12$ & 1.50 & $55 \cdot 19$ & $1 \cdot 22$ & 0.0014 \\
\hline NHIII & $20-59$ & SRTT & 50.91 & $1 \cdot 15$ & $47 \cdot 25$ & $2 \cdot 31$ & 49.23 & 1.97 & $51 \cdot 34$ & 1.64 & 50.85 & 1.68 & 0.7903 \\
\hline $\mathrm{NHIII}$ & $20-59$ & 20-59 All & $49 \cdot 87$ & $0 \cdot 72$ & $48 \cdot 62$ & 1.05 & $50 \cdot 11$ & 1.40 & $50 \cdot 96$ & $1 \cdot 27$ & $52 \cdot 92$ & 0.90 & 0.0061 \\
\hline NHIII & $60+$ & SR-1 & 47.61 & 0.85 & 54.44 & 2.55 & $48 \cdot 17$ & 1.28 & 54.52 & $2 \cdot 11$ & $51 \cdot 22$ & $2 \cdot 01$ & 0.0020 \\
\hline $\mathrm{NHIII}$ & $60+$ & SR-2 & $47 \cdot 01$ & $0 \cdot 70$ & $53 \cdot 29$ & $2 \cdot 24$ & $48 \cdot 45$ & 1.51 & 54.55 & $1 \cdot 82$ & 49.81 & $1 \cdot 79$ & 0.0001 \\
\hline $\mathrm{NHIII}$ & $60+$ & SR-All & $47 \cdot 31$ & $0 \cdot 74$ & $53 \cdot 87$ & $2 \cdot 32$ & $48 \cdot 31$ & 1.32 & 54.53 & 1.89 & $50 \cdot 51$ & $1 \cdot 80$ & 0.0003 \\
\hline NH 99-02 & $60+$ & DSST & $48 \cdot 39$ & $1 \cdot 22$ & $51 \cdot 08$ & $1 \cdot 25$ & $53 \cdot 32$ & 1.47 & $50 \cdot 27$ & 1.60 & $53 \cdot 28$ & 1.93 & 0.0743 \\
\hline
\end{tabular}

SDLT, serial digit learning task; SDST, symbol-digit substitution test; SRTT, simple reaction time task; SR-1, story recall one; SR-2, story recall two; DSST, digit-symbol substitution test.

${ }^{*}$ Data were adjusted for sex, ethnicity, age, smoking status, energy intake $(\mathrm{kJ}), \mathrm{BMl}\left(\mathrm{kg} / \mathrm{m}^{2}\right)$, poverty:income ratio, intakes of saturated fat $(\mathrm{g})$, monounsaturated fat (g), polyunsaturated fat $(\mathrm{g})$, cholesterol $(\mathrm{mg})$, carbohydrate $(\mathrm{g})$ and protein $(\mathrm{g})$.

this information will probably result in a better understanding of not only dairy-specific associations but also of nutrientcognition interactions.

The present data also indicated that there may be a threshold effect of dairy product consumption in the $60+$ age group. Moving from a non-consumer or low-consumer to the level of an average consumer (approximately two servings per $\mathrm{d}$ ) seemed to have a greater effect on the cognitive assessments than moving beyond two servings of dairy products. However, more information is needed before this finding can be verified. Very recently, a cross-sectional analysis of dairy product consumption and cognitive measures utilising data from the Maine-Syracuse Longitudinal Study was published ${ }^{(27)}$. In this analysis, consumption of dairy products at least once per $\mathrm{d}$ was associated with an improvement in several measures of cognitive function. However, a similar threshold effect was observed, albeit at a lower level, indicating that in order to see an effect of dairy product consumption, a strict accounting of baseline dairy product intake may be necessary.

The lack of an association with milk in the present analysis is also interesting since our hypothesis was that all dairy products should have a similar effect on cognition and some previous studies have indicated an association with milk ${ }^{(2,28)}$. This potential association seems to be even more logical since fortified milk contributes a significant amount of vitamin D to the diet, and several studies have highlighted the potential contribution of vitamin D to cognitive function ${ }^{(11,29-31)}$. However, the exact mechanism for the ability of vitamin $\mathrm{D}$ to regulate cognition is still unknown and no clinical interventions with dietary vitamin D have established causality. The association between milk and cognition may be related to the type of milk being consumed (e.g. full fat $v$. non-fat). The present study did not disassociate between full-fat and reduced-fat milk and therefore may have missed an association, since other studies have shown relationships with low-fat varieties $^{(15)}$. Future studies should track not only dairy products, but also individual dairy products, when examining cognitive endpoints, in an effort to determine which products or components within those products may be having the greatest effect on learning and memory.

Since the present study indicated that milk consumption had no association with cognition while cheese consumption $\mathrm{did}$, it is difficult to speculate on the potential mechanism of action. It is possible that during the process of cheese development, bioactive compounds are formed which could have effects on memory. These compounds would need to be small, since the blood-brain barrier could prevent the access of complex molecules to critical areas of action. It is also possible that by providing a higher amount of certain protein components, lipids, vitamins or minerals than milk in a single serving, cheese more aptly replenishes the CNS with critical nutrients. For example, cheese may provide specific amino acids such as cysteine that are critical for glutathione production in a higher amount than milk ${ }^{(32)}$. Having adequate amounts of cysteine available for glutathione production during periods of inflammatory stress could allow the CNS to regulate any inflammation that arises and maintain homeostasis. In addition, providing lipids to the CNS so that neuronal cells and their signalling matrices are functioning optimally could be a secondary mechanism of action. The phospholipid components of the milk fat globule membrane, which is found in all fat-containing dairy products, may be one source of these necessary nutrients. Prior research has indicated that sphingolipids, which are found in the milk fat globule membrane, can dramatically affect neuronal growth and development ${ }^{(33)}$. However, their role in the maintenance of cognition is still not known. It is also possible that the consumption of other foods associated with cheese consumption (e.g. red wine), not adjusted for in the present analysis, may have also influenced the results, since these foods may contain compounds that have been suggested to affect cognition ${ }^{(34)}$. In addition, cheese intake may be an indicator of socioeconomic status and although we attempted to normalise this effect by adjusting for the poverty:income ratio, it is possible that the observed association could be driven by better general health. Once a better understanding of the role that 
specific nutrients and foods have in cognition is obtained, greater adjustment for these covariates can be utilised.

As an intervention to avoid deficits in cognition as the population ages, subtle changes in dietary intake are an attractive proposition. These changes could have dramatic effects on the loss of social functioning and lessen the economic impact of cognitive diseases without requiring too dramatic a shift in behaviour. In addition, nutrients or other bioactive compounds that are determined to have even a small effect via consumption may be very important to the development of more potent nutraceuticals or pharmaceuticals. As more information on food and cognitive function becomes available, it may be possible to tailor dietary advice for individuals at a significant risk for CNS-centric health issues. The present data indicate that more research on the ability of certain foods, such as cheese, to affect cognition is certainly needed.

\section{Acknowledgements}

This study was funded by the Dairy Research Institute. The authors' contributions are as follows: K. M. P. designed the project and developed the manuscript; V. L. F performed the data analysis/modelling and edited the final manuscript. Conflicts of interest: K. M. P. is an employee of the Dairy Research Institute. V. L. F. received research funding from the Dairy Research Institute.

\section{References}

1. von Arnim CA, Gola U \& Biesalski HK (2010) More than the sum of its parts? Nutrition in Alzheimer's disease. Nutrition 26, 694-700

2. Lee L, Kang SA, Lee HO, et al. (2001) Relationships between dietary intake and cognitive function level in Korean elderly people. Public Health 115, 133-138.

3. Iadecola C (2010) The overlap between neurodegenerative and vascular factors in the pathogenesis of dementia. Acta Neuropathol 120, 287-296.

4. Gorelick PB, Scuteri A, Black SE, et al. (2011) Vascular contributions to cognitive impairment and dementia: a statement for healthcare professionals from the American Heart Association/American Stroke Association. Stroke 42, 2672-2713.

5. Corsinovi L, Biasi F, Poli G, et al. (2011) Dietary lipids and their oxidized products in Alzheimer's disease. Mol Nutr Food Res 55, Suppl. 2, S161-S172.

6. Kalmijn S, Launer LJ, Ott A, et al. (1997) Dietary fat intake and the risk of incident dementia in the Rotterdam Study. Ann Neurol 42, 776-782.

7. Engelhart MJ, Geerlings MI, Ruitenberg A, et al. (2002) Diet and risk of dementia: Does fat matter?: The Rotterdam Study. Neurology 59, 1915-1921.

8. Engelhart MJ, Geerlings MI, Ruitenberg A, et al. (2002) Dietary intake of antioxidants and risk of Alzheimer disease. JAMA 287, 3223-3229.

9. Luchsinger JA, Tang MX, Miller J, et al. (2007) Relation of higher folate intake to lower risk of Alzheimer disease in the elderly. Arch Neurol 64, 86-92.

10. Kado DM, Karlamangla AS, Huang MH, et al. (2005) Homocysteine versus the vitamins folate, $\mathrm{B} 6$, and $\mathrm{B} 12$ as predictors of cognitive function and decline in older high-functioning adults: MacArthur Studies of Successful Aging. Am J Med 118, $161-167$.
11. Pogge E (2010) Vitamin D and Alzheimer's disease: is there a link? Consult Pharm 25, 440-450.

12. Barberger-Gateau P, Raffaitin C, Letenneur L, et al. (2007) Dietary patterns and risk of dementia: the Three-City cohort study. Neurology 69, 1921-1930.

13. Scarmeas N, Stern Y, Mayeux R, et al. (2009) Mediterranean diet and mild cognitive impairment. Arch Neurol 66 , $216-225$

14. Scarmeas N, Stern Y, Tang MX, et al. (2006) Mediterranean diet and risk for Alzheimer's disease. Ann Neurol 59 912-921

15. Camfield DA, Owen L, Scholey AB, et al. (2011) Dairy constituents and neurocognitive health in ageing. Br J Nutr (epublication ahead of print version 22 February 2011).

16. Crichton GE, Bryan J, Murphy KJ, et al. (2010) Review of dairy consumption and cognitive performance in adults: findings and methodological issues. Dement Geriatr Cogn Disord 30, 352-361.

17. Proctor SP, Letz R \& White RF (2000) Validity of a computerassisted neurobehavioral test battery in toxicant encephalopathy. Neurotoxicology 21, 703-714.

18. Mahoney FC, Moore PA, Baker EL, et al. (1988) Experimental nitrous oxide exposure as a model system for evaluating neurobehavioral tests. Toxicology 49, 499-457.

19. Zhang J, McKeown RE, Muldoon MF, et al. (2006) Cognitive performance is associated with macronutrient intake in healthy young and middle-aged adults. Nutr Neurosci 9, 179-187.

20. Yaffe K, Lindquist K, Shlipak MG, et al. (2008) Cystatin C as a marker of cognitive function in elders: findings from the health ABC study. Ann Neurol 63, 798-802.

21. Rosano C, Simonsick EM, Harris TB, et al. (2005) Association between physical and cognitive function in healthy elderly: the health, aging and body composition study. Neuroepidemiology 24, 8-14.

22. Fabrigoule C, Rouch I, Taberly A, et al. (1998) Cognitive process in preclinical phase of dementia. Brain 121, 135-141.

23. Anonymous (1996) Plan and Operation of the Third National Health and Nutrition Examination Survey (NHANES III, 1988-1994), 1996 ed. Hyattsville, MD: National Center for Health Statistics.

24. Anonymous (1996) National Health and Nutrition Examination Survey (NHANES): MEC Interviewers Procedures Manual. Atlanta, GA: Centers for Disease Control and Prevention

25. Rahman A, Sawyer Baker P, Allman RM, et al. (2007) Dietary factors and cognitive impairment in community-dwelling elderly. J Nutr Health Aging 11, 49-54.

26. Astrup A, Dyerberg J, Elwood P, et al. (2011) The role of reducing intakes of saturated fat in the prevention of cardiovascular disease: where does the evidence stand in 2010? Am J Clin Nutr 93, 684-688.

27. Crichton GE, Elias MF, Dore GA, et al. (2012) Relation between dairy food intake and cognitive function: The Maine-Syracuse Longitudinal Study. Int Dairy J 22, 15-23.

28. Yamada M, Kasagi F, Sasaki H, et al. (2003) Association between dementia and midlife risk factors: the Radiation Effects Research Foundation Adult Health Study. J Am Geriatr Soc 51, 410-414.

29. Llewellyn DJ, Lang IA, Langa KM, et al. (2010) Vitamin D and risk of cognitive decline in elderly persons. Arch Intern Med 170, 1135-1141.

30. Llewellyn DJ, Lang IA, Langa KM, et al. (2011) Vitamin D and cognitive impairment in the elderly U.S. population. J Gerontol A Biol Sci Med Sci 66, 59-65. 
31. Seamans KM, Hill TR, Scully L, et al. (2010) Vitamin D status and measures of cognitive function in healthy older European adults. Eur J Clin Nutr 64, 1172-1178.

32. Sekhar RV, Patel SG, Guthikonda AP, et al. (2011) Deficient synthesis of glutathione underlies oxidative stress in aging and can be corrected by dietary cysteine and glycine supplementation. Am J Clin Nutr 94, 847-853.
33. Buccoliero R \& Futerman AH (2003) The roles of ceramide and complex sphingolipids in neuronal cell function. Pharmacol Res 47, 409-419.

34. Tiwari V \& Chopra K (2011) Resveratrol prevents alcoholinduced cognitive deficits and brain damage by blocking inflammatory signaling and cell death cascade in neonatal rat brain. J Neurochem 117, 678-690. 